\title{
PROPELLER PERFORMANCE IN PRESENCE OF FREESTREAM
}

\author{
Mojtaba Hedayatpour, Mehran Mehrandezh \\ Faculty of Engineering and Applied Science \\ University of Regina \\ Regina, Canada \\ \{hedayatm,mehran.mehrandezh\}@uregina.ca
}

\author{
Farrokh Janabi-Sharifi \\ Department of Mechanical and Industrial Engineering \\ Ryerson University \\ Toronto, Canada \\ fsharifi@ryerson.ca
}

\begin{abstract}
This paper presents mathematical modeling for thrust force and moments generated by a propeller. In particular, the effects of freestream on propeller's performance are investigated. We introduce some of the applications of the proposed model in modeling multi-rotor UAVs which helps to increase stability or maneuverability of the vehicle. In the end, simulation results for thrust force and moments of an example propeller in presence of a uniform freestream are presented.
\end{abstract}

Keywords-component; propeller; aerodynamics; thrust force; unmanned aerial vehicles, freestream

\section{INTRODUCTION (HEADING 1)}

Multi-rotors have gained significant attention in recent years. Due to their simplicity and maneuverability, they have been used in a broad spectrum of applications such as bioengineering [1], agronomy [2], sports [3] and inspection of infrastructures [4].

In modeling of UAVs, aerodynamic model of propellers plays an important role as it determines the majority of forces and moments in the system. Therefore, an accurate model for the propellers is crucial in analyzing such a system. In the literature, typically, the thrust force and moment generated by the propeller is assumed to be proportional to the square of its angular velocity and the effects of freestream on its performance is usually neglected by assuming small freestream velocities [5], [6], [7]. However, this model is not valid in high speed flight and its accuracy deteriorates as flight speed increases [8], [9].

The freestream may affect propeller's performance depending on its direction and magnitude. Generally, these effects could change propeller's performance in two ways: i) changing the effective angle of attack of the blades; and ii) changing the local airflow velocity over the blades. The former effect is caused when there is a freestream with its velocity vector parallel to the angular velocity vector of the propeller while the latter is caused by any freestream with its velocity vector perpendicular to the angular velocity vector of the propeller. Studying these effects not only helps us to derive a more realistic mathematical model for multi-rotors but also helps to find more stable and power-optimal configurations for such vehicles. In this paper we use Blade Element Theory (BET) to calculate the thrust force and moment of each blade element.

The paper is organized as follows: Section II presents the effects of freestream on propeller's performance along with simulation results for thrust force and moments of an example propeller. Finally, the paper concludes in Section III followed by presenting some of the applications of the proposed model in multi-rotor UAV research.

\section{EFFECTS OF FREESTREAM ON PROPELLER'S PERFORMANCE}

\section{A. Notation}

The term ${ }^{I} \boldsymbol{\omega}_{p}$ denotes that $\boldsymbol{\omega}$ belongs to $p$ and is expressed in frame $I$. Angular velocity vector of the vehicle is represented by $\omega_{B}=(p, q, \mathrm{r})^{T}$ where $p, q$ and $r$ are roll, pitch and yaw rates respectively. Also, 2-Norm of $\omega$ is represented by $\|\omega\|$ and absolute value of $s$ is shown by $|s|$.

\section{B. Effects of Freestream with Its Velocity Vector}

Perpendicular to Angular Velocity Vector of the Propeller

Suppose we have a propeller turning at angular velocity $\omega_{p}$ as expressed in a frame attached to its COM as shown in Fig. 1. The propeller has two blades of radius $R_{b}$ and is assumed to have constant chord $c$. For simplicity, first, we assume there is an almost uniform freestream with velocity vector $V_{\infty_{1}}$, as shown in blue in Fig. 1, which is parallel to the y-axis.

Consider a blade element (small hashed area in Fig. 2) of length $c$ and differential width $d r_{b}$ where $r_{b}$ is the distance of the blade element from the root of the blade. As shown in Fig. 2 , the rotation of the blade generates relative air flow velocity with magnitude $r_{b}\left\|\omega_{p}\right\|$, over each blade element. As the propeller is turning, the relative air flow velocity over the blade element could either be increased or decreased depending on the azimuth angle of the blade and direction of the freestream velocity. The azimuth angle $\psi_{p}$ is defined as the angle between 
the blade and the direction of $V_{\infty_{1}}$. Therefore, the resultant relative air flow velocity over each blade element can be written as:

$$
v=r_{b}\left\|\omega_{p}\right\|+\left\|V_{\infty_{1}}\right\| \sin \psi_{p}
$$

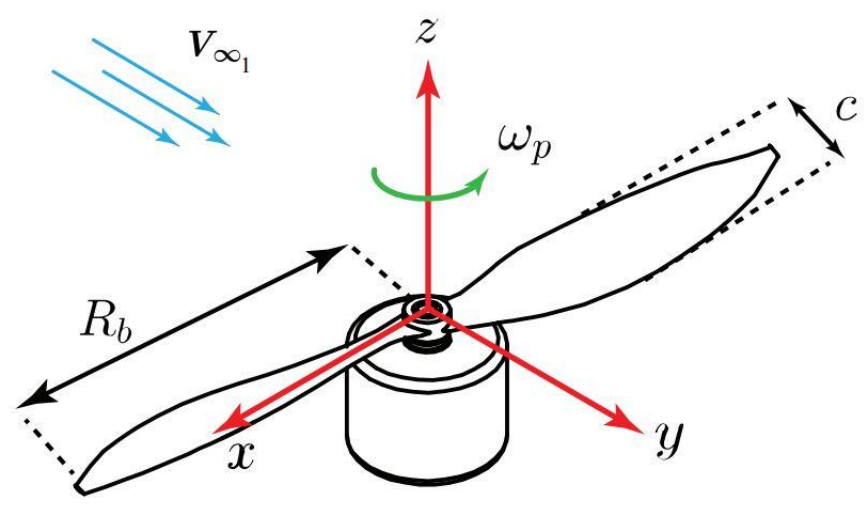

Figure 1: Schematic of a propeller with a frame attached to its COM.

It can be seen in Fig. 2, for the advancing blade $\left(0 \leq \psi_{p} \leq \pi\right)$, freestream velocity increases the relative air flow velocity over the blade and for the retreating blade ( $\left.\pi \leq \omega_{p} \leq 2 \pi\right)$, it decreases the relative air flow velocity. The changes in the relative air flow velocity with azimuth angle affects the overall thrust of the propeller and it generates a moment in the direction of the freestream velocity as shown in blue.

Therefore, using (1) and according to Blade Element Theory, thrust force and moments of each blade element can be calculated as follows:

$$
\begin{gathered}
d f_{p}=\frac{1}{2} \rho_{a} c C_{L} v^{2} d r_{b} \\
d \tau_{d p}=\frac{1}{2} \rho_{a} c C_{D} v^{2} r_{b} d r_{b} \\
d \tau_{p}=\frac{1}{2} \rho_{a} c C_{L} v^{2} r_{b} \sin \psi_{p} d r_{b}
\end{gathered}
$$

where $\rho_{a}$ represents air density and $C_{L}$ and $C_{D}$ are the lift and drag coefficient of the airfoil of the blade respectively. Also, $f_{p}$ represents thrust force of the blade element, $\tau_{d_{p}}$ represents the moment due to drag force of the blade element and $\tau_{p}$ represents the moments due to change in thrust force with respect to the azimuth angle of the blade.
By integrating (2), (3) and (4) over blade radius $r_{b}$ and azimuth angle $\psi_{p}$, average thrust force and the average moments for one blade as functions of freestream velocity and angular velocity of the propeller can be calculated as follows:

$$
\begin{gathered}
f_{p}=\frac{1}{2} \rho_{a} c C_{L}\left(\frac{2 R_{b}^{3}}{3}\left\|\omega_{p}\right\|^{2}+\left\|V \infty_{1}\right\|^{2} R_{b}\right) \\
\tau_{d_{p}}=\frac{1}{4} \rho_{a} c C_{D}\left(R_{b}^{4}\left\|\omega_{p}\right\|^{2}+\left\|V \infty_{1}\right\|^{2} R_{b}^{2}\right) \\
\tau_{p}=\frac{1}{2} \rho_{a} c C_{L} R_{b}^{3}\left\|\omega_{p}\right\|\left\|V_{\infty_{1}}\right\|
\end{gathered}
$$

Note that by assuming zero freestream velocity, equations (5), (6) and (7) yield the simplified model for thrust force and moments of a propeller which is widely used in the literature (i.e., [3], [10], [11], [12]).

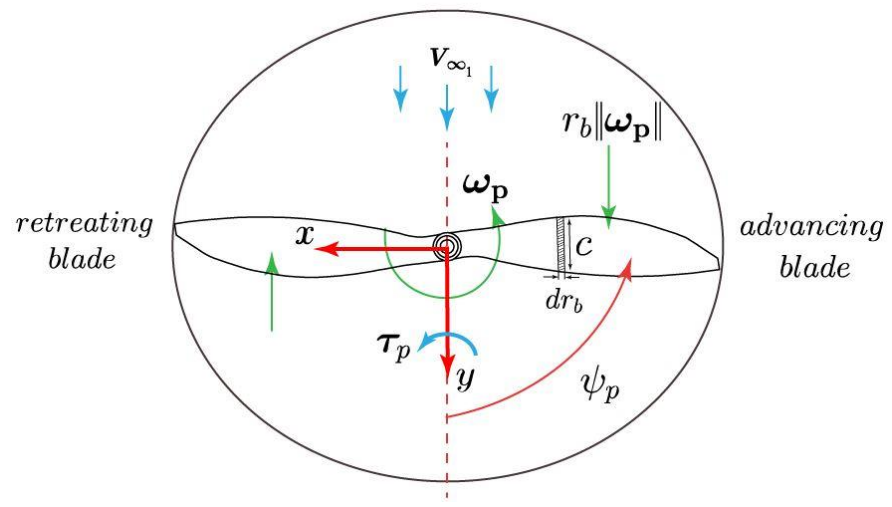

Figure 2: A propeller in presence of freestream.

Using the proposed model, simulation results for two complete turns of a propeller with angular velocity $\left\|\omega_{p}\right\|=900 \mathrm{rad} / \mathrm{s}$ in presence of freestream velocity is presented in Fig. 3. Note that the direction of rotation and freestream velocity are the same as those in Fig. 2. The remaining parameters of the simulations are as follows: $c=0.03 \mathrm{~m}$, $C_{L}=1.022, C_{D}=0.01, R_{b}=0.08 \mathrm{~m}$ and $\rho_{a}=1.225 \mathrm{~kg} / \mathrm{m}^{3}$. In Fig. 3, the top plot presents variations of thrust force with respect to blade azimuth. The red color represents the thrust force when $\left\|V_{\infty_{1}}\right\|=0$, which is constant, meaning that the relative air flow velocity over the blade element is constant for all azimuth angles. The blue color, represents thrust force of the propeller as a function of azimuth angle when freestream velocity is nonzero, $\left\|V_{\infty_{1}}\right\|=10 \mathrm{~m} / \mathrm{s}$. Comparing both scenarios, it can be seen that for nonzero freestream velocity and for $0 \leq \psi_{p} \leq \pi$ thrust force is increased while for $\pi \leq \omega_{p} \leq 2 \pi$ it is decreased which is due to higher relative air flow velocities on the advancing blade than that over the retreating blade. The 
yellow color shows the average of thrust force of the propeller when $\left\|V_{\infty_{1}}\right\|=10 \mathrm{~m} / \mathrm{s}$. Results show that in presence of nonzero freestream velocity the average thrust force of the propeller increases.
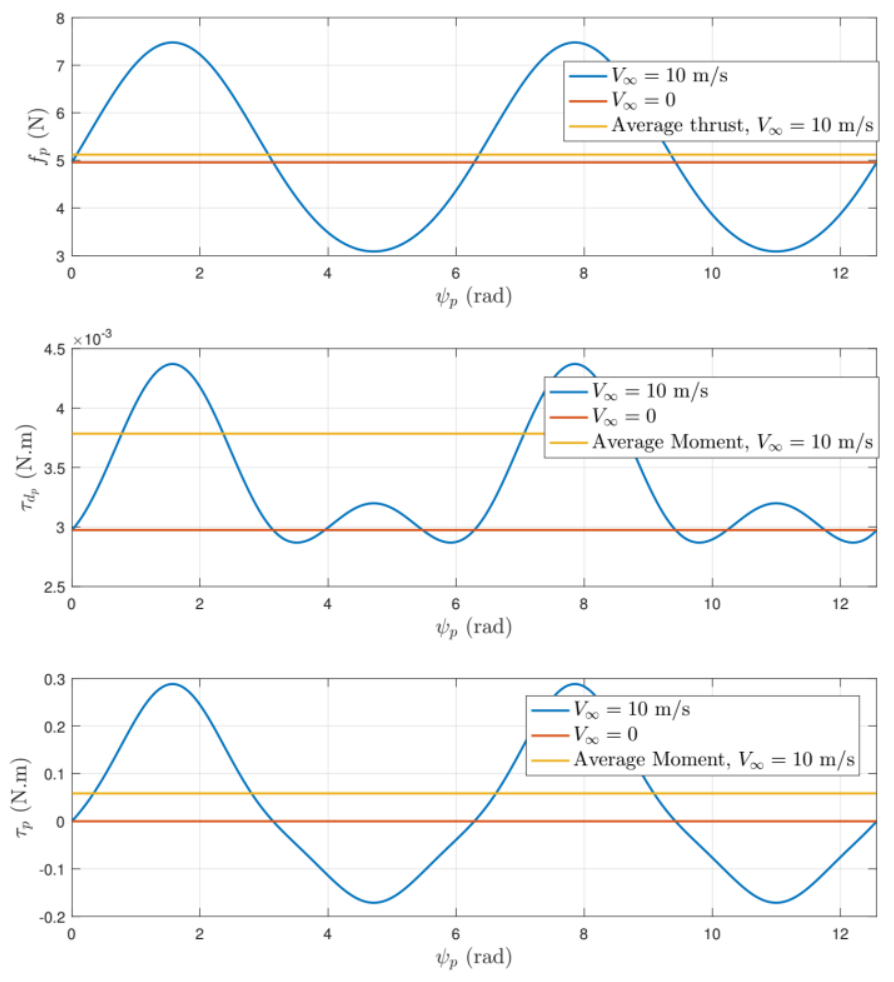

Figure 3: Simulation results for thrust force and moments of a propeller in presence of freestream.

The middle and bottom plots in Fig. 3 present variations of the moments due to drag and freestream $\tau_{d_{p}}$ and $\tau_{p}$ ) versus azimuth angle respectively. The red color represents the moment when $\left\|V_{\infty_{1}}\right\|=0 \mathrm{~m} / \mathrm{s}$, blue color represents variations of moment in presence of freestream velocity $\left\|V_{\infty_{1}}\right\|=10 \mathrm{~m} / \mathrm{s}$ and the yellow color represents the average moment of the propeller when $\left\|V_{\infty_{1}}\right\|=10 \mathrm{~m} / \mathrm{s}$.

\section{Effects of Freestream with Its Velocity Vector in Parallel with Angular Velocity Vector of the Propeller}

We continue investigating the effects of freestream on propeller's performance. However, as shown in Fig. 4, this time we assume the freestream velocity vector, $V_{\infty_{2}}$, is parallel to the angular velocity vector of the propeller, $\omega_{p}$, as expressed in the frame attached to it.

Suppose the propeller is turning at angular velocity $\omega_{p}$ as shown in Fig. 5. In absence of freestream, there will be an airflow velocity vector $r_{b} \omega_{p}$ over each blade element as shown in green. Also, for each blade element, the angle of attack $(\mathrm{AOA}) \Theta$ is defined as the angle between the chord of the blade element and the local airflow velocity vector $r_{b} \omega_{p}$.

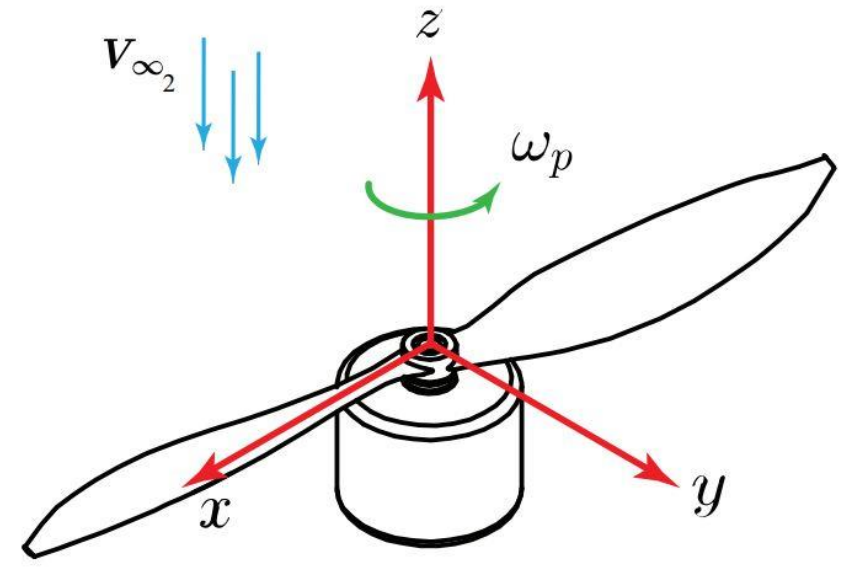

Figure 4: Propeller in presence of freestream. The freestream velocity vector $V_{\infty_{2}}$ is assumed to be parallel with the angular velocity vector of the propeller $\omega_{p}$.

Now, consider an almost uniform freestream with velocity vector $V_{\infty_{2}}$ in the positive direction of z-axis as shown in blue in Fig. 5 top. It can be seen that such a freestream changes the direction and magnitude of the resultant airflow velocity over the blade element as shown in red in Fig. 5 top. Therefore, the new angle of attack $\Theta^{\prime}$, in presence of freestream is greater than that in absence of freestream $\left(\Theta^{\prime} \geq \Theta\right)$.

However, if the freestream velocity vector is in the negative direction of z-axis (see Fig. 5 bottom), it changes the direction and magnitude of the resultant airflow velocity vector such that it decreases the effective angle of attack $\left(\Theta^{\prime} \leq \Theta\right)$.
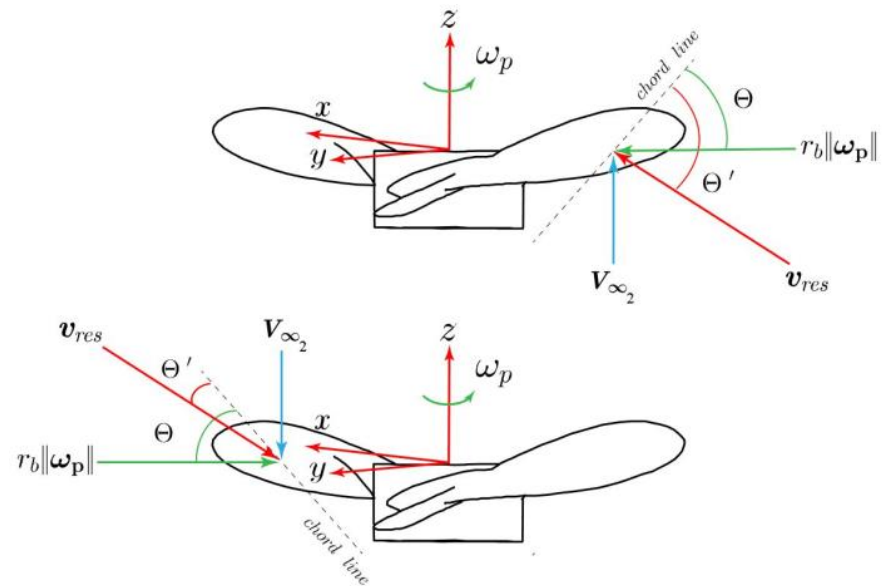

Figure 5: Propeller in presence of freestream. The freestream velocity vector $V_{\infty_{2}}$ is assumed to be parallel with $\omega_{p}$. On top, $V_{\infty_{2}}$ is the positive direction of z-axis and in bottom it is assumed to be in the opposite direction.

The importance of studying AOA is because it directly affects the lift coefficient of the blade element and consequently affects the thrust force generated by the propeller. At low speed flight (subsonic) and assuming small angles, the lift coefficient $C_{L}$ changes almost linearly with AOA which can be written as follows [13]: 


$$
\frac{\Delta C_{L}}{\Delta \Theta}=\sigma
$$

where $\sigma$ is a constant which is determined through experiments in wind tunnel. From (2), we know that any increase (decrease) in $C_{L}$ increases (decrease) the thrust force of the blade element. Therefore, one can summarize the results as follows:

- Any freestream with positive (negative) z-component velocity (expressed in the propeller's frame) increases (decreases) the AOA which increases (decreases) the thrust force.

Furthermore, to formulate the changes in thrust force of the propeller, first we can write the changes in AOA of each blade element as follows:

$$
\Delta \Theta=\Theta-\Theta^{\prime}=\arctan \frac{\left\|V_{\infty_{2}}\right\|}{\left\|r_{b} \omega_{p}\right\|}
$$

Finally, using (2), (8) and (9), the changes in thrust force of the propeller can be written as follows:

$$
\Delta f_{p}=\frac{1}{4} \rho_{a} c \sigma R_{b}^{2}\left\|V_{\infty_{2}}\right\|\left\|\omega_{p}\right\|
$$

From (10), it can be seen that the changes in thrust force is proportional to the magnitude of the freestream velocity vector $V_{\infty_{2}}$.

Table I presents the parameters involved in the modeling.

TABLE I. PROPELler PARAMETERS

\begin{tabular}{|c|c|}
\hline$\omega_{p}$ & angular velocity vector of the propeller \\
\hline$\Theta$ & angle of attack \\
\hline$C_{L}$ and $C_{D}$ & lift and drag coefficients of the airfoil \\
\hline$V_{\infty}$ & freestream velocity vector \\
\hline$\sigma$ & slope of $C_{L}$ vs $C_{D}$ curve for the airfoil \\
\hline$R_{b}$ & blade radius of the propeller \\
\hline$c$ & chord of the blade \\
\hline$\rho_{a}$ & air density \\
\hline
\end{tabular}

\section{CONCLUSIONS}

In summary, we presented the significance of the effects of freestream on performance of propellers. We formulated these effects as functions of propeller parameters and also the parameters of the freestream. Table I, presents all parameters involved in the proposed propeller model.
The proposed propeller model may be used in modeling multi-rotor UAVs. Such a model is used in quadcopters where it is shown that by tilting the rotors by a small angle as shown in Fig. 6, more stable or maneuverable configurations can be found [8]. The model is also used in modeling spinning-type multi-rotor UAVs to find optimal-power hover solutions. Fig. 7 , presents a monospinner UAV with a single propeller where the spinning fuselage generates a freestream over the propeller [9].

For the future work, we plan to compare the proposed model with experimental results from experiments in wind tunnel for a particular propeller and investigate the accuracy and shortcomings of the model.

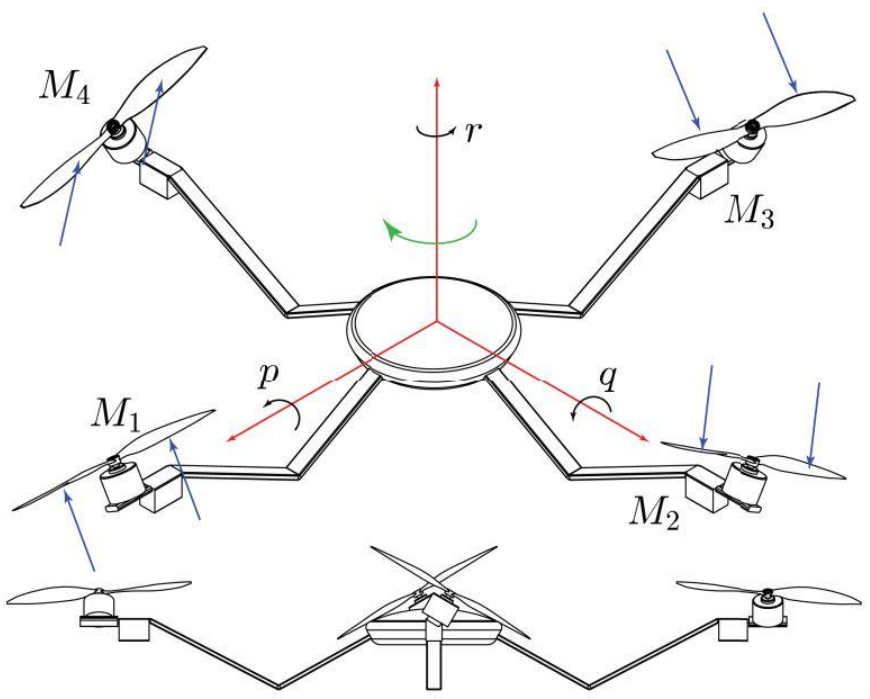

Figure 6: A quadcopter with tilted rotors. The blue arrows shows the direction of freestream velocity on each propeller as expressed in the body frame (red).

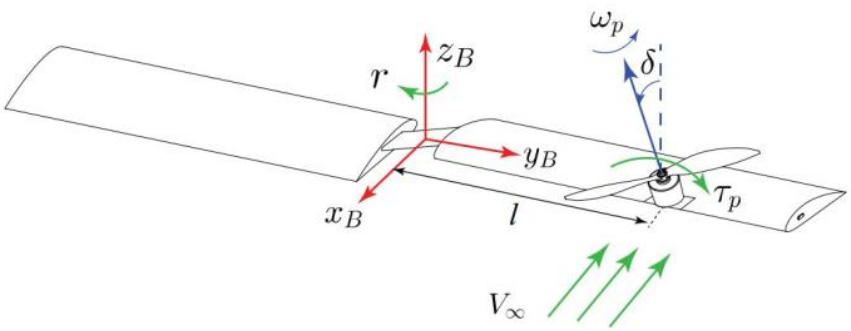

Figure 7: A monospinner UAV with tilted rotor. As the fuselage is spinning with yaw rate $r$, the propeller experiences a freestream, as shown in green.

\section{REFERENCES}

[1] J. Thomas, J. Polin, K. Sreenath, , and V. Kumar, "Avian-inspired grasping for quadcopter micro uavs," Bioinspiration \& Biomimetics, vol. 9, pp. 25010-25019,2014

[2] J. Rasmussen, J. Nielsen, F. Garcia-Ruiz, S. Christensen, and J. C. Streibig, "Potential uses of small unmanned aircraft systems (uas) in weed research," Weed Research, vol. 4, no. 53, pp. 242\{248, 2013.

[3] M. Muller, S. Lupashin, and R. D'Andrea, "Quadrocopter ball juggling," in 2017 IEEE/RSJ International Conference on Intelligent Robots and Systems (IROS), 2011, pp. 5113-5120. 
[4] L. F. Luque-Vega, B. Castillo-Toledo, A. Loukianov, and L. E. Gonzalez-Jimenez, "Power line inspection via an unmanned aerial system based on the quadrotor helicopter," in MELECON 2014 - 17th IEEE Mediterranean Electrotechnical Conference, April 2014, pp. 393397.

[5] M. Y. Amir and V. Abbass, "Modeling of quadrotor helicopter dynamics," in International Conference on Smart Manufacturing Application, 2008, pp. 100-105.

[6] D. Mellinger, N. Michael, and V. Kumar, "Trajectory generation and control for precise aggressive maneuvers with quadrotors," The International Journal of Robotics Research, vol. 31, no. 5, pp. 664-674, 2012.

[7] J. Wang, T. Bierling, M. Achtelik, L. Hocht, F. Holzapfel, W. Zhao, and G. Hiong, "Attitude free position control of a quadcopter using dynamic inversion," in AIAA Infotech@ Aerospace, 2011, pp. 29-31.

[8] M. Hedayatpour, M. Mehrandezh, and F. Janabi-Sharifi, "A unified approach to configuration-based dynamic analysis of quadcopters for optimal stability," in 2017 IEEE/RSJ International Conference on Intelligent Robots and Systems (IROS), Sept 2017, pp. 5116-5121.

[9] M. Hedayatpour, M. Mehrandezh, and F. Janabi-Sharifi, "Revised propeller dynamics and energy-optimal hovering in a monospinner," in The 4th International Conference of Control, Dynamic Systems, and Robotics, August 2017, pp. 1-8.

[10] M. W. Mueller and R. D'Andrea, "Relaxed hover solutions for multicopters: application to algorithmic redundancy and novel vehicles," International Journal of Robotics Research, vol. 35, no. 8, pp. 873-889, 2015.

[11] M. W. Mueller and R. D'Andrea, "Stability and control of a quadrocopter despite the complete loss of one, two, or three propellers," in 2014 IEEE International Conference on Robotics and Automation (ICRA), 2014, p. 4552.

[12] M. Hehn and R. D'Andrea, "Quadrocopter trajectory generation and control," in 18th IFAC World Congress, Milano, 2011, pp. 1485-1491.

[13] J. D. Anderson, Fundamentals of Aerodynamics, 5th ed. United States: McGraw Hill Higher Education, 2016. 